\title{
Measurement of effective blast energy for direct initiation of spherical gaseous detonations from high-voltage spark discharge
}

\author{
Bo Zhang ${ }^{1}$, Hoi Dick $\mathrm{Ng}^{2 *}$, John H.S. Lee ${ }^{3}$ \\ ${ }^{1}$ Beijing Institute of Technology \\ State Key Laboratory of Explosion Science and Technology, Beijing, 100081, China \\ ${ }^{2}$ Concordia University \\ Department of Mechanical and Industrial Engineering \\ Montreal, H3G 1M8, Canada \\ ${ }^{3}$ McGill University \\ Department of Mechanical Engineering, Montreal, H3A 2K6, Canada \\ *Corresponding Author \\ Department of Mechanical and Industrial Engineering \\ Concordia University \\ 1455 de Maisonneuve Blvd. West \\ Montreal, H3G 1M8, Canada \\ e-mail: hoing@encs.concordia.ca \\ Tel.: (514) 848-2424 (ext. 3177) \\ Fax: (514) 848-3175
}

Final revision submitted to Shock Waves Journal

October, 2011 


\title{
Measurement of effective energy for direct initiation of spherical gaseous detonations from high-voltage spark discharge
}

Bo Zhang, Hoi Dick Ng and John H.S. Lee

\begin{abstract}
In this study, effective energy from spark discharge for direct blast initiation of spherical gaseous detonations is investigated. In the experiment, direct initiation of detonation is achieved via a spark discharge from a high voltage and low inductance capacitor bank and the spark energy is estimated from the analysis of the current output. To determine the blast wave energy from the powerful spark, the time-of-arrival of the blast wave in air is measured at different radii using a piezoelectric pressure transducer. Good agreement is found in the scaled blast trajectories, i.e., scaled time $c_{\mathrm{o}} \cdot t / R_{\mathrm{O}}$ where $c_{\mathrm{o}}$ is the ambient sound speed, as a function of blast radius $R_{\mathrm{S}} / R_{\mathrm{O}}$ between the numerical simulation of a spherical blast wave from a point energy source and the experimental results where the explosion length scale $R_{\mathrm{o}}$ is computed using the equivalent spark energy from the first $1 / 4$ current discharge cycle. Alternatively, by fitting the experimental trajectories data, the blast energy estimated from the numerical simulation appears also in good agreement with that obtained experimentally using the $1 / 4$ cycle criterion. Using the $1 / 4$ cycle of spark discharge for the effective energy, direct initiation experiments of spherical gaseous detonations are carried out to determine the critical initiation energy in $\mathrm{C}_{2} \mathrm{H}_{2}-2.5 \mathrm{O}_{2}$ mixtures with $70 \%$ and $0 \%$ argon dilution. The experimental results obtained from the $1 / 4$ cycle of spark discharge agree well with the prediction from two initiation models, namely, the Lee's surface energy model and a simplified work done model. The main source of discrepancy in the comparison can be explained by the uncertainty of cell size measurement which is needed for both the semi-empirical models.
\end{abstract}

Keywords: direct initiation; spherical detonation; critical energy; blast energy; spark ignition 


\section{Introduction}

Since the pioneering work by Zel'dovich et al. [1], the problem of direct blast initiation of spherical detonation has been extensively studied [2]. Direct blast initiation refers to the instantaneous formation of the detonation without going through the pre-detonation stage of flame acceleration. By "instantaneous" it means that the initial strong blast wave is generated directly by the ignition source upon the rapid deposition of its energy and decays asymptotically to a Chapman-Jouguet (CJ) detonation rather than by flame acceleration, as in the transition from deflagration to detonation (DDT) [3, 4]. Among different dynamic parameters of detonation waves, the minimum energy required for the direct blast initiation of a detonation in a given explosive mixture (i.e., the critical initiation energy) has been considered as perhaps the most direct means of determining an explosive sensitivity [5].

Experimentally, direct initiation can be achieved by electrical discharges, high power lasers, and condensed explosive charges. Studies have indicated that condensed explosive charges or sparks from a rapid low inductance capacitor discharge can closely approximate the ideal instantaneous point energy source of the classical similarity blast wave solution $[6,7]$.

For the spark discharge initiation, earlier investigation suggested the total energy stored in the capacitors $E_{\mathrm{c}}$ (i.e., $E_{c}=1 / 2 C V^{2}$, in joules, where $C$ denotes the capacitance, in farads; and $V$ the potential difference, in volts) could be used for the estimation for critical energy of direct initiation. However, owing to the energy loss and ohmic dissipation this kind of estimate cannot correctly evaluate the total energy deposited into the explosive and thus, the actual energy responsible for the initiation of detonations. Therefore, it only gives an order of magnitude approximation to the actual energy deposited in the mixtures. 
Knystautas et al. [8] have shown in their study that the actual spark discharge energy can be adequately estimated by integrating the square of current function $i(t)^{2}$ multiplied by the spark

resistance $R_{\mathrm{sp}}: E_{\text {total }}=\int_{0}^{\infty} i^{2} R_{\mathrm{sp}} d t$. Experimental observation also indicated that the detonation is usually formed in a time scale $t_{\mathrm{e}}$ which is much less than the total spark discharge time and thus, the energy deposited subsequent to $t_{\mathrm{e}}$ plays no significant role in the initiation process since the initiation is already completed. Further analysis also suggests that the effective spark energy for direct initiation corresponds to the first $1 / 4$ cycle of the current discharge, but evidences are lacking to confirm this hypothesis.

The objective of the present study is two fold: First, the experimentally measured blast decay in air from the spark discharge is first compared with the numerical simulation of a point blast. This attempts to verify if the effective spark energy from the $1 / 4$ cycle of current discharge is equivalent to the point blast energy in the ideal case. Second, the critical energy, determined using the $1 / 4$ cycle spark discharge energy estimation, for direct initiation of spherical detonation in stoichiometric acetylene-oxygen mixtures with $70 \%$ and $0 \%$ argon dilution at different initial pressures are reported. The experimental results are then compared with theoretical prediction using the Lee's surface energy model and a simplified work done model.

\section{Experimental Details and Measurement}

The time-of-arrival measurement of the blast front decay originated from the spark is carried out in the experimental setup illustrated in Fig. 1. The slender coaxial electrode for spark discharge is fixed at the bottom of a hemisphere by a steel holder; a PCB piezoelectric pressure transducer \# PCB 401A22 (with sensitivity of $3.0965 \mathrm{mV} / \mathrm{kPa}$ from our laboratory calibration and typical rise time of $\sim 2 \mu \mathrm{s}$ ) is mounted in a horizontal plate above the spark to measure the arrival time 
of the spark-generated blast wave. Two vertical steel plates are fixed to support the horizontal plate. The distance between the spark and the transducer can be changed via adjusting the position of the horizontal plate.

The ignition system is constructed in previous studies (see $[9,10]$ for further details). It essentially consists of a high voltage power supply, capacitor bank, a gap-switch and a trigger module (TM-11A). At the end of this slender electrode there is a $3.5 \mathrm{~mm}$ spark gap through which the energy is delivered through the ignition circuit. The procedure to estimate the actual spark discharge energy from the ignition system is also detailed in authors' previous studies $[9$, 10]. For completeness, it is briefly described here (see $[9,10]$ for a complete analysis). For a damped oscillatory discharge the current is modeled by a function $i(t)=A e^{-\alpha . t} \sin (\omega t)$ with the attenuation factor equal to $\alpha=R_{\text {total }} / 2 L_{\text {total }}$ where $R_{\text {total }}$ and $L_{\text {total }}$ are the total circuit resistance and inductance, respectively. For a given trace of the current discharge recorded on the oscilloscope, the natural frequency $\omega_{n}$ is determined using frequency of the discharge $\omega$ and the damping factor $\zeta$ where $\omega=\sqrt{1 /(L C)_{\text {total }}-(R / 2 L)_{\text {total }}^{2}}=\omega_{n} \sqrt{1-\varsigma^{2}}$. The total circuit inductance and subsequently the total circuit resistance are then calculated using the natural frequency $\omega_{n}$ and total capacitance $C_{\text {total }}$ from $L_{\text {total }}=1 / \omega_{n}^{2} C_{\text {total }}$ and $R_{\text {total }}=2 L_{\text {total }} \alpha$, respectively. The spark resistance $R_{\mathrm{sp}}$ is determined by subtracting $R_{\mathrm{total}}=R_{\text {circuit }}$ when the spark gap is shorted out $R_{\mathrm{sp}} \approx 0$ from $R_{\text {total }}=R_{\text {circuit }}+R_{\mathrm{sp}}$ when the spark is not shorted. At last, the total spark discharge energy is found by numerically integrating the square of the current $i$ multiplied by spark resistance $R_{\mathrm{sp}}$ : $E_{\text {total }}=\int_{0}^{\infty} i^{2} R_{s p} d t$. Table 1 summarizes details of the typical experimental parameters and results of spark discharge for different initial voltage. $R_{\text {circuit }}$ and $R_{\mathrm{sp}}$ represent the resistance of circuit and spark, respectively. Again, the energy $E_{c}=1 / 2 C V^{2}$ means the total energy stored on the 
capacitor. Owing to the ohmic dissipation and energy loss, only part of this stored total energy contribute to the total spark discharge energy (i.e., $\left.E_{\text {total }}\right)$.

\section{Numerical Simulation}

To compare the experimental blast decay measurement and to determine the blast energy, numerical simulation of blast wave propagation from a point source is performed. The full unsteady nonlinear dynamics of blast wave decay is modeled numerically by solutions of the inviscid compressible, time-dependent one-dimensional Euler equations, which are expressed as the conservation equations for mass, momentum and energy:

$$
\begin{gathered}
\frac{\partial \rho}{\partial t}+\frac{\partial(\rho u)}{\partial r}+\frac{2}{r}(\rho u)=0 \\
\frac{\partial(\rho u)}{\partial t}+\frac{\partial\left(\rho u^{2}+p\right)}{\partial r}+\frac{2}{r}\left(\rho u^{2}\right)=0 \\
\frac{\partial(\rho e)}{\partial t}+\frac{\partial u(\rho e+p)}{\partial r}+\frac{2}{r}[u(\rho e+p)]=0
\end{gathered}
$$

where $t$ is the time and $r$ is the radial coordinate. $\rho$ denotes the mass density, $u$ the velocity, $p$ the pressure, and $e$ the specific total energy with specific heat ratio $\gamma$.

$$
e=\frac{p}{(\gamma-1) \rho}+\frac{1}{2} u^{2}
$$

The variables are all normalized with the initial quiescent state of the medium, i.e., initial density, initial pressure, sound speed in ambient air $c_{\mathrm{o}}$ as the reference flow variables. The solution of the above system is approximated numerically using two hydrodynamic codes based on two different numerical schemes to ensure that the numerical formulation does not affect the blast trajectories, particularly at low Mach number regime. The two numerical finite-volume methods, i.e., the Slope-Limiter Centered (SLIC) scheme and the upwind Weighted-Average 
Flux (WAF) scheme with an exact Riemann solver, are both conservative, explicit, second order accurate schemes [11]. A value of 0.8 is used for the CFL number and two resolutions are checked for the simulations $\Delta r=0.02$ and 0.01 to ensure that the solution is grid-independent. To initialize the computation, the strong blast wave profiles $[12,13]$ are used as initial conditions at initial normalized blast radius $R_{s}=2$ (the reference scale is chosen such that there is 100 numerical grid points per unit length), and Mach number $M=40$.

For comparison, the blast wave trajectories from both experiments and numerical simulations are scaled with respect to the explosion length $R_{\mathrm{o}}$ as a characteristic length scale defined as:

$$
R_{0}=\left(\frac{E_{s}}{p_{o}}\right)^{1 / 3}
$$

where $E_{\mathrm{s}}$ denotes the blast wave energy and $p_{o}$ the initial pressure $\left(p_{o}=101.3 \mathrm{kPa}\right.$ in the case of the present experiment). For the numerical simulation, the blast wave energy $E_{\mathrm{s}}$ can be obtained from the initial strong blast profile. The experimental explosion length $R_{\mathrm{o}}$ is computed using the $1 / 4$ cycle spark discharge energy as the effective blast energy for comparison and validation.

\section{Results and Discussion}

The variation of scaled time $c_{0} . t / R_{\mathrm{o}}$ where $c_{\mathrm{o}}$ is the ambient sound speed, as a function of spherical blast wave radius $R_{\mathrm{s}} / R_{\mathrm{o}}$ determined numerically and experimentally by the time-ofarrival data obtained from the pressure transducer is plotted in Fig. 2. The numerical results obtained using the two numerical schemes, and using SLIC with two numerical grid resolutions, are plotted in this same figure and one can see that there is hardly any difference between all these numerical solutions. By comparing the numerical solution with the experimental data, one can see that both agree well and follow the same trend. Within the range of measurement, the maximum discrepancy between the numerical results and the experimental data is about $10 \%$ 
occurred at the further $R_{\mathrm{s}} / R_{0}$, which appears acceptable taking into account the experimental limitation and the flow sensitivity at the low Mach number regime (experimental data points at the largest $R_{\mathrm{S}} / R_{\mathrm{o}}$ corresponds to a Mach number $\sim 1.15$ ). Recalled that the experimental results are all scaled with the characteristic explosion length computed using $1 / 4$ cycle spark discharge energy as the effective blast energy and therefore, this good agreement thus confirms that the $1 / 4$ cycle spark energy can be used adequately to represent the actual energy contributing to the blast wave generation.

For better comparison without any scaling, using the trajectories data we can also fit with the numerical solution to determine the blast energy. Figure 3 shows the numerical blast decay solution (of a particular point source energy $E_{(\text {numerical) }}$ ) that matches with the dimensional experimental data. This blast energy determined from the matching is compared with the $1 / 4$ cycle energy in Table 1. On average for all experimental conditions, the \% difference is found to be around $20 \%$. It is worth noting that the $1 / 4$ cycle spark energy measurement for each experiment has a variation or a maximum uncertainty of $\pm 8 \%$. Taking this experimental limitation into account, the energies from both the numerical estimation and the experimental measurement appear to be in reasonably good agreement.

The above result provides evidences to confirm that the $1 / 4$ cycle energy is equivalent to the effective blast energy. In fact, for direct initiation via a spark discharge, it is long considered that only this $1 / 4$ cycle of the energy discharge contributes to the direct initiation process [8]. As discussed earlier, the estimation of total spark energy is from the function of:

$$
E_{\text {total }}=\int_{0}^{t} i^{2} R_{s p} d t
$$

where $i(t)=A e^{-\alpha \cdot t} \sin (\omega t)$ and spark resistance $R_{\mathrm{sp}}$ is constant. Knystautas et al. [8] defined peak average power $P=E_{\text {total }} / t$, henceforth the critical energy for direct initiation as the total energy 
released to the mixture up to maximum of the peak average power $P=\left(E_{\text {total }} / t\right)_{\max }$ (where the maximum strength of the shock wave occurs when $P$ is a maximum). It is suggested that the time up to peak average power as well as the maximum strength of the shock wave is approximately the first quarter cycle of the discharge; energy release after first cycle peak plays little role in its subsequent motion, since it is the chemical energy released by the exothermic reactions initiated by the shock wave itself that sustains its subsequent propagation. Long time energy release only tends to heat up the spark channel and does not contribute to the blast wave for direct initiation.

Using the assumption of $1 / 4$ cycle spark discharge energy to estimate the point blast energy for the direct initiation, initiation experiments are carried out in the same high pressure bomb [9, $10]$ and results are compared with Lee's surface energy theoretical model $[6,14]$ and a simplified work done model $[5,15,16]$.

Lee's surface energy model is a simple semi-empirical phenomenological model that relates the point blast initiation mode with the planar wave initiation mode and the critical energy is correlated with the cell size as a characteristic length scale. The link is established based on the minimum surface energy of the critical tube to the surface area of the critical size of the minimum detonation kernel, in other words, the correlation is through equating the surface energy contained in the wave in both cases at criticality. Thus the surface energy contained in the point blast initiated spherical detonation wave at the time when the wave has decayed to the CJ state from its overdriven state at a distance $R^{*}$ is equivalent to the energy in the planar detonation wave in the problem of critical tube diameter, $d_{c}$. Hence equating the minimum surface areas of both waves at criticality:

$$
4 \pi R^{* 2}=\left(\frac{\pi}{4}\right) d_{c}^{2}
$$


thus obtained a kernel radius $R^{*}=\frac{1}{4} d_{c}$. From strong blast wave theory, the blast wave energy is given by:

$$
E_{s}=4 \pi I \gamma p_{o} M_{s}^{2} R_{s}^{3}
$$

where $p_{\mathrm{o}}$ is ambient pressure, $M_{\mathrm{S}}$ the blast Mach number and $R_{\mathrm{S}}$ is the blast radius. $I$ is an numerical constant (for specific heat ratio $\gamma=1.4, I=0.423$ ) [3]. According to Zel'dovich's criterion [1], when the blast wave decays to $M_{\mathrm{s}}=M_{\mathrm{CJ}}$, it should engulf a kernel size of radius $R_{S}=R^{*}$

A simplified work done model is also considered in this study for comparison $[5,15,16]$. In essential, if we assume the energy needed to re-initiate a detonation downstream of the unconfined space in the critical tube diameter problem can be related to the work done delivered by the detonation product in the confined tube (i.e., a fictitious piston) over a period $t^{*}$, the energy can be obtained by:

$$
E_{s}=\int_{0}^{t^{*}} p_{C J} \frac{\pi d_{c}^{2}}{4} u_{C J} d t
$$

where $p_{\mathrm{CJ}}$ and $u_{\mathrm{CJ}}$ denote the $\mathrm{CJ}$ detonation pressure and particle velocity, respectively. $t^{*}$ can be modeled as the time when the rarefaction wave reaches the tube axis, which can be approximately by $t^{*} \sim d_{\mathrm{c}} / 2 c_{C J}$ with $c_{\mathrm{CJ}}$ being the sound speed of the detonation products $[5,17]$. Therefore, this simplified work done model gives:

$$
E_{s}=\frac{p_{C J} u_{C J} \pi d_{c}^{3}}{8 c_{C J}}
$$

To complete both the Lee's initiation model and the simplified work done model, the value of critical tube diameter, $d_{\mathrm{c}}$, is required for the energy estimation. According to Lee, the critical tube diameter and failure are closely linked to the reaction sensitivity of the mixture and hence, 
the detonation cellular instability [18]. As shown in previous studies [10, 19-21], for the stable argon-diluted mixtures like $\mathrm{C}_{2} \mathrm{H}_{2}-2.5 \mathrm{O}_{2}-70 \%$ in that the reaction zone is at least piecewise laminar described by the ZND model and cellular instabilities play a minor role on the detonation propagation, the empirical correlation of the critical tube diameter with the characteristic detonation cell size $d_{\mathrm{c}} \sim 25-30 \lambda$ can generally be used. Our recent experimental study also confirms that for $\mathrm{C}_{2} \mathrm{H}_{2}-2.5 \mathrm{O}_{2}-70 \%, d_{\mathrm{c}}=29 \lambda[22]$; and therefore, the energy equations for both models immediately yield:

$$
\begin{aligned}
& E_{s}=4 \pi I p_{o} M_{C J}^{2}\left(\frac{d_{c}}{4}\right)^{3} \cong 1525 \pi \rho_{o} V_{C J}^{2} I \lambda^{3} \quad \text { Surface energy model } \\
& E_{s}=\frac{p_{C J} u_{C J} \pi d_{c}^{3}}{8 c_{C J}}=\frac{24389 p_{C J} u_{C J} \pi \lambda^{3}}{8 c_{C J}} \quad \text { Simplified work done model }
\end{aligned}
$$

where $\rho_{\mathrm{o}}$ is the initial density of the mixture, $V_{\mathrm{CJ}}, p_{\mathrm{CJ}}, u_{\mathrm{CJ}}, c_{\mathrm{CJ}}$ the $\mathrm{CJ}$ detonation velocity, pressure, particle velocity and sound speed, $\lambda$ the cell size and $I$ is a numerical constant (for $\gamma=$ 1.4, $I=0.423)$. All the equilibrium $\mathrm{CJ}$ detonation properties are computed from the thermodynamic equilibrium program CEA [23]). On the other hand, for undiluted $\mathrm{C}_{2} \mathrm{H}_{2}-2.5 \mathrm{O}_{2}$ mixture in which cellular instabilities plays a prominent role in the propagation mechanism, the empirical correlation is universally given by $d_{c}=13 \lambda[24,25]$ and this yields both the energy equations to become:

$$
\begin{aligned}
& E_{s}=4 \pi \gamma p_{o} M_{C J}^{2} I\left(\frac{13 \lambda}{4}\right)^{3}=\frac{2197}{16} \pi \rho_{o} V_{C J}^{2} I \lambda^{3} \quad \text { Surface energy model } \\
& E_{s}=\frac{p_{C J} u_{C J} \pi d_{C}^{3}}{8 c_{C J}}=\frac{2197 p_{C J} u_{C J} \pi \lambda^{3}}{8 c_{C J}} \quad \text { Simplified work done model }
\end{aligned}
$$

Values of cell size $\lambda$ with initial pressure for the two acetylene-oxygen mixture conditions are obtained from $[26,27]$. The cell sizes for the Ar-diluted mixture are obtained directly by 
Radulescu [26] and for the undiluted case, the correlation is determined from different data obtained in the CALTECH detonation database [27] (a compilation from Desbordes et al. [21, 28], Laberge et al. [29], Voitsekhovskii et al. [30] and Knystautas et al. [25] for the pressure range of interest in this study). These correlations are given as:

$$
\begin{aligned}
& \lambda[\mathrm{mm}]=a \cdot\left(p_{o}[\mathrm{kPa}]\right)^{b} \quad \text { where } a=18.9, b=-1.015 \text { undiluted } 0 \% \mathrm{Ar} \\
& \lambda[\mathrm{mm}]=a \cdot\left(p_{o}[\mathrm{kPa}]\right)^{b} \quad \text { where } a=113.8, b=-1.20 \text { diluted } 70 \% \mathrm{Ar}
\end{aligned}
$$

Results of critical energy for $\mathrm{C}_{2} \mathrm{H}_{2}-2.5 \mathrm{O}_{2}$ mixture with $70 \%$ of argon dilution initial pressure from $50 \mathrm{kPa}$ to $200 \mathrm{kPa}$ are plotted in Fig. 4. The plot shows the critical initiation energy for direct initiation of a spherical detonation obtained from the theoretical prediction, using both Lee's surface energy model and the simplified work done model, and experiments as a function of initial pressure. The experimental curve is obtained through least-square regression in view of better comparison. It can be seen that good agreement is found between the $1 / 4$ cycle spark discharge energy and the two model predictions which are developed semi-empirically and phenomenologically.

Similarly, Fig. 5 shows the plot for the undiluted acetylene-oxygen mixtures. It summarizes the experiment results and compared with theoretical ones for $\mathrm{C}_{2} \mathrm{H}_{2}-2.5 \mathrm{O}_{2}$ mixture with initial pressure ranging from $5 \mathrm{kPa}$ to $20 \mathrm{kPa}$. In general, all curves show the same trend. However, a large discrepency can been seen as the the initial pressure decreases. It is worth noting that for undiluted mixture, the cell size is usually irregular at low initial pressure, making the determination of a characteristic cell size difficult. It is well known that the uncertainty in the cell size data for unstable mixtures can be differed as much as a factor of 2. Figure 6 shows the cell size data used to construct the correlation given in Eq. (13-14) and one can notice the scattering at low initial pressure. The presence of such amibiguity in cell size measurement thus 
has a large effect on the energy estimation using any theoretical model that relies on the value of detonation cell size due to the cubic dependency as given in Eq. (11-14). Within the inherent uncertainty in the cell size data, the result indeed shows a fair agreement between the $1 / 4$ cycle energy measurement and the theoretical predictions.

\section{Concluding Remarks}

In the present study, we investigated the effective critical energy for direct blast initiation of spherical gaseous detonations from a powerful spark. This work first compared the numerical simulation of blast wave decay from a point source with experimental measurement to show that the effective amount of blast energy can be estimated by the $1 / 4$ cycle from the spark discharge. Using the effective energy of the $1 / 4$ cycle spark discharge, we report in this paper the critical energy of direct initiation of spherical gaseous detonation for $\mathrm{C}_{2} \mathrm{H}_{2}-2.5 \mathrm{O}_{2}$ mixtures with $70 \%$ and $0 \%$ argon dilution at different initial pressures and compare with two semi-empirical models. The critical energies for direct initiation from a spark as determined by the amount of $1 / 4$ cycle of spark discharge agree well with the theoretical predictions from both Lee's surface energy and a simplified work done model. Such good agreement indirectly further validates the effective critical energy from the $1 / 4$ cycle of spark discharge can be linked to the energy responsible for direct initiation and analogous to the point blast energy in the ideal case.

\section{Acknowledgments}

BZ thanks the Chinese Scholarship Council (CSC) for the granting of a fellowship for research at McGill University. This work is supported by the Natural Sciences and Engineering Research Council of Canada (NSERC). 


\section{References}

1. Zel'dovich, Y.B., Kogarko, S.M., Simonov, N.N.: An experimental investigation of spherical detonation in gases. Sov. Phys. Tech. Phys. 1, 1689-1713 (1957).

2. Lee, J.H.S., Higgins, A.J.: Comments on Criteria for Direct Initiation of Detonation. Phil. Trans. R. Soc. Lond. A 357, 3503-3521 (1999).

3. Lee, J.H.: Initiation of Gaseous Detonation. Ann. Rev. Phys. Chem 28, 75-104 (1977).

4. Lee, J.H.: Dynamic parameters of gaseous detonations. Ann. Rev. Fluid. Mech. 16, 311336 (1984).

5. Matsui, H. and Lee, J.H.S.: On the measure of the relative detonation hazards of gaseous fuel-oxygen and air mixtures. Proc. Combust. Inst. 17, 1269-1280 (1978).

6. Benedick, W.B., Guirao, C.M., Knystautas, R., Lee, J.H.S.: Critical charge for the direct initiation of detonation in gaseous fuel-air mixtures. Prog. Astronaut. Aeronaut. 106, 181-202 (1986).

7. Zitoun, R., Desbordes, D., Guerraud, C., Deshaies, B.: Direct initiation of detonation in cryogenic gaseous $\mathrm{H}_{2}-\mathrm{O}_{2}$ mixtures. Shock Waves 4(6) 331-337 (1995).

8. Knystautas, R., Lee, J.H.S.: On the effective energy for direct initiation of detonations. Combust. Flame 27, 221-228 (1976).

9. Kamenskihs, V., Ng, H.D., Lee, J.H.S.: Measurement of critical energy for direct initiation of spherical detonations in high-pressure $\mathrm{H}_{2}-\mathrm{O}_{2}$ mixtures. Combust. Flame, 157(9), 1795-1799 (2010).

10. Zhang, B., Kamenskihs, V., Ng, H.D., Lee, J.H.S.: Direct blast initiation of spherical gaseous detonation in highly argon diluted mixtures. Proc. Combust. Inst. 33 (2), 2265$2271(2010)$. 
11. Toro, E.F: Riemann Solvers and Numerical Methods for Fluids Dynamics. $2^{\text {nd }}$ edition, Springer-Verlag, 1999.

12. Sedov, L.I.: Similarity and Dimensional Methods in Mechanics, $10^{\text {th }}$ edition, CRC Press, Boca Raton, FL, 1993.

13. Taylor, G.I.: The formation of a blast wave by a very intense explosion: I. Theoretical discussion. Proc. R. Soc. Lond. A 201, 159-174 (1950).

14. Lee, J.H.S., Knystautas, R., Guirao, C.M.: The link between cell size, critical tube diameter, initiation energy and detonability limits. In: Lee, J.H.S., Guirao, C.M. (Eds.), Fuel-Air Explosions, University of Waterloo Press 157-187 (1982).

15. Desbordes, D.: Transmission of overdriven plane detonations: critical diameter as a function of cell regularity and size. Prog. Astro. Aero. 11, 170-185 (1988).

16. Sochet, I., Lamy, T., Brossard, J., Vaglio, C., Cayzac, R.: Critical tube diameter for detonation transmission and critical initiation energy of spherical detonation. Shock Waves 9, 113-123 (1999).

17. Vasil'ev A.A.: Diffraction estimate of the critical energy for initiation of gaseous detonation. Combust. Expl. Shock Waves, 34(4), 433-437 (1998).

18. Lee, J.H.S.: On the critical tube diameter. In: J. Bowen, Editor, Dynamics of Exothermicity, Gordon and Breach, Amsterdam, Netherlands, 321-335 (1996).

19. Moen, I., Sulmistras, A., Thomas, G.O., Bjerketvedt, D., Thibault, P.A.: Influence of cellular regularity on the behavior of gaseous detonations. Prog. Astronaut. Aeronaut. 106, 220-243 (1986).

20. Shepherd, J.E., Moen, I., Murray, S., Thibault, P.A.: Analyses of the cellular structure of detonations. Proc. Combust. Inst. 21, 1649-1658 (1986). 
21. Desbordes, D., Guerraud, C., Hamada, L., Presles, H.N.: Failure of the classical dynamic parameters relationships in highly regular cellular detonation systems. Prog. Astronaut. Aeronaut. 153, 347-359 (1993).

22. Zhang, B., Ng, H.D., Lee, J.H.S.: Direct measurement and relationship between critical tube diameter and critical energy for direct detonation initiation. $23^{\text {rd }}$ Int. Colloquium on the Dynamics of Explosions \& Reactive Systems, Irvine, US, July 24-July 29, 2011.

23. McBride, B.J., Gordon, S.: Computer program for calculation of complex chemical equilibrium compositions and applications II, Users manual and program description. NASA Rep. RP-1311-P2, 1996.

24. Mitrofanov, V.V., Soloukhin, R.I.: The diffraction of multi-front detonation waves. Soy. Phys.-Dokl. 9, 1055-1058 (1965).

25. Knystautas, R., Lee, J.H., Guirao, C.: The critical tube diameter for detonation failure in hydrocarbon-air mixtures. Combust. Flame 48, 63-83 (1982).

26. Radulescu, M.I.: The Propagation and Failure Mechanism of Gaseous Detonations: Experiments in Porous-Walled Tubes. Ph.D. thesis, McGill University, Canada, (2003).

27. Kaneshige, M., Shepherd, J.E.: Detonation Database. Explosion Dynamics Laboratory Report FM97-8 July 30, (1997).

28. Desbordes, D.: Transmission of overdriven plane detonations: Critical diameter as a function of cell regularity and size. Prog. Astronaut. Aeronaut. 114, 170-185 (1988).

29. Laberge, S., Knystautas, R., Lee, J.H.S.: Propagation and extinction of detonation waves in tube bundles. Prog. Astronaut. Aeronaut. 153, 381-396 (1993).

30. Voitsekhovskii, B.V., Mitrofanov, V.V., Topchian, M.E.: The structure of a detonation front in gases. Technical Report FTD-MT-64-527, Wright-Patterson AFB, 1966. 


\section{Table}

Table 1. Experimental parameters and results for spark discharge

\begin{tabular}{ccccccccc}
\hline $\begin{array}{c}\text { Voltage } \\
(\mathrm{KV})\end{array}$ & $\begin{array}{c}C \\
(\mu \mathrm{F})\end{array}$ & $\begin{array}{c}R_{\text {circuit }} \\
(\Omega)\end{array}$ & $\begin{array}{c}R_{\mathrm{sp}} \\
(\Omega)\end{array}$ & $\begin{array}{c}E_{c}=1 / 2 \mathrm{CV}^{2} \\
(\mathrm{~J})\end{array}$ & $\begin{array}{c}E_{\text {total }} \\
(\mathrm{J})\end{array}$ & $\begin{array}{c}E_{1 / 4 \text { Cycle }} \\
(\mathrm{J})\end{array}$ & $\begin{array}{c}E_{(\text {Numerical })} \\
(\mathrm{J})\end{array}$ & $\begin{array}{c}\text { Comparison } \\
(\%)\end{array}$ \\
\hline 18 & 2 & 0.1142 & 0.0131 & 324 & 27.78 & 6.00 & 5.22 & 87.0 \\
16 & 2 & 0.1164 & 0.0125 & 256 & 21.52 & 4.78 & 4.08 & 85.4 \\
14 & 2 & 0.1268 & 0.0119 & 196 & 16.76 & 3.72 & 2.88 & 77.4 \\
12 & 2 & 0.1323 & 0.0101 & 144 & 13.12 & 2.73 & 2.15 & 78.8 \\
\hline
\end{tabular}

Table 1. 


\section{Figure Captions}

Fig. 1 Sketch of blast wave TOA measurement setup

Fig. 2 Scaled blast trajectories of $c_{\mathrm{o}} . t / R_{\mathrm{o}}$ as a function of $R_{\mathrm{S}} / R_{\mathrm{o}}$

Fig. 3 Blast energy estimation from the numerical solutions for different wave trajectories

Fig. 4 Critical initiation energy for direct initiation of a spherical detonation in $\mathrm{C}_{2} \mathrm{H}_{2}-2.5 \mathrm{O}_{2}$ mixture with $70 \%$ of argon as a function of initial pressure with $d_{\mathrm{c}}=29 \lambda$

Fig. 5 Critical initiation energy for direct initiation of a spherical detonation in $\mathrm{C}_{2} \mathrm{H}_{2}-2.5 \mathrm{O}_{2}$ mixture as a function of initial pressure with $d_{\mathrm{c}}=13 \lambda$

Fig. 6 Cell size data from $[21,25,27-30]$ and the correlation for $\mathrm{C}_{2} \mathrm{H}_{2}-2.5 \mathrm{O}_{2}$ mixture as a function of initial pressure 


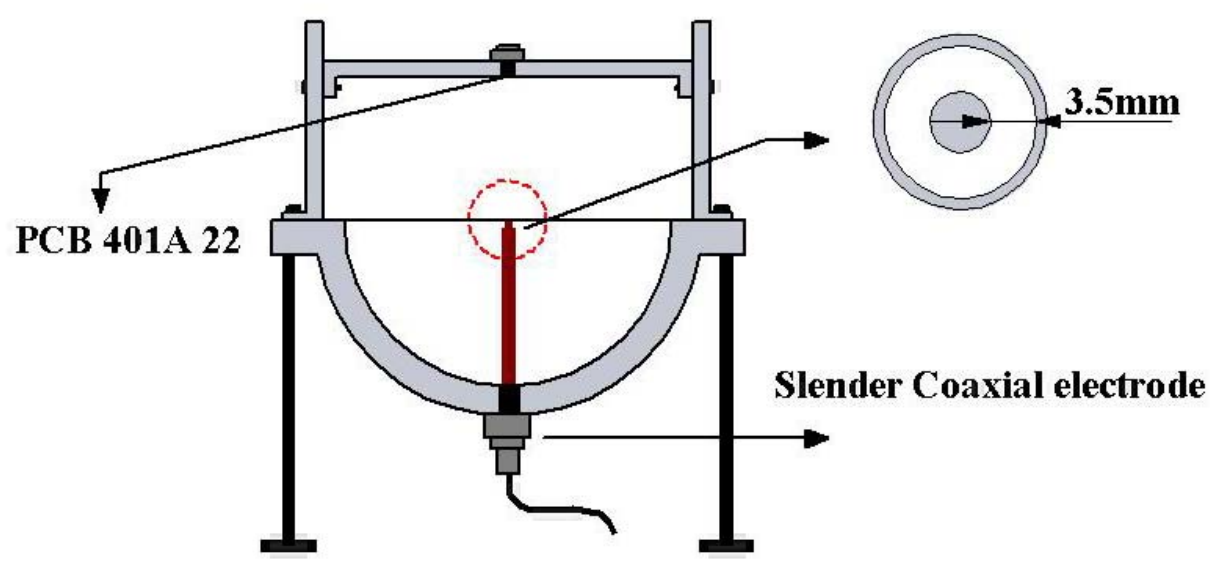

Fig. 1 


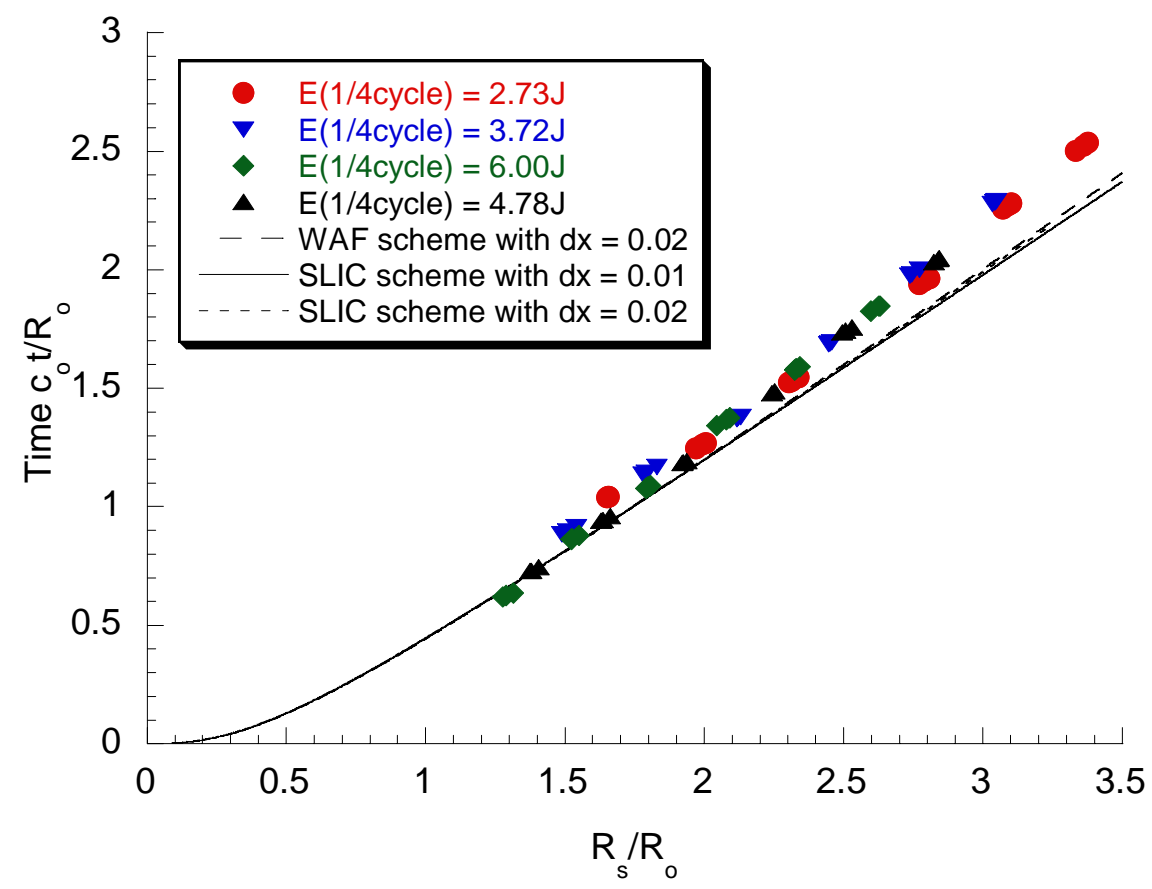

Fig. 2 


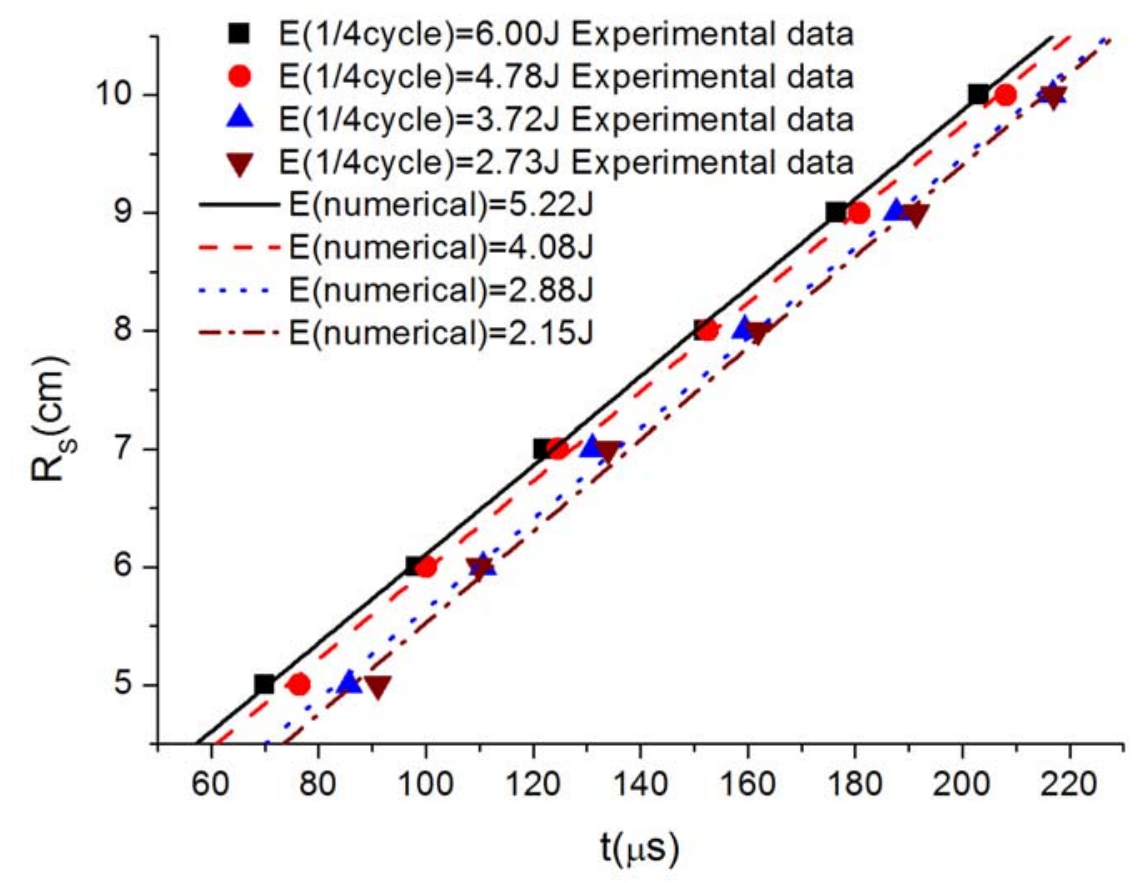

Fig. 3 


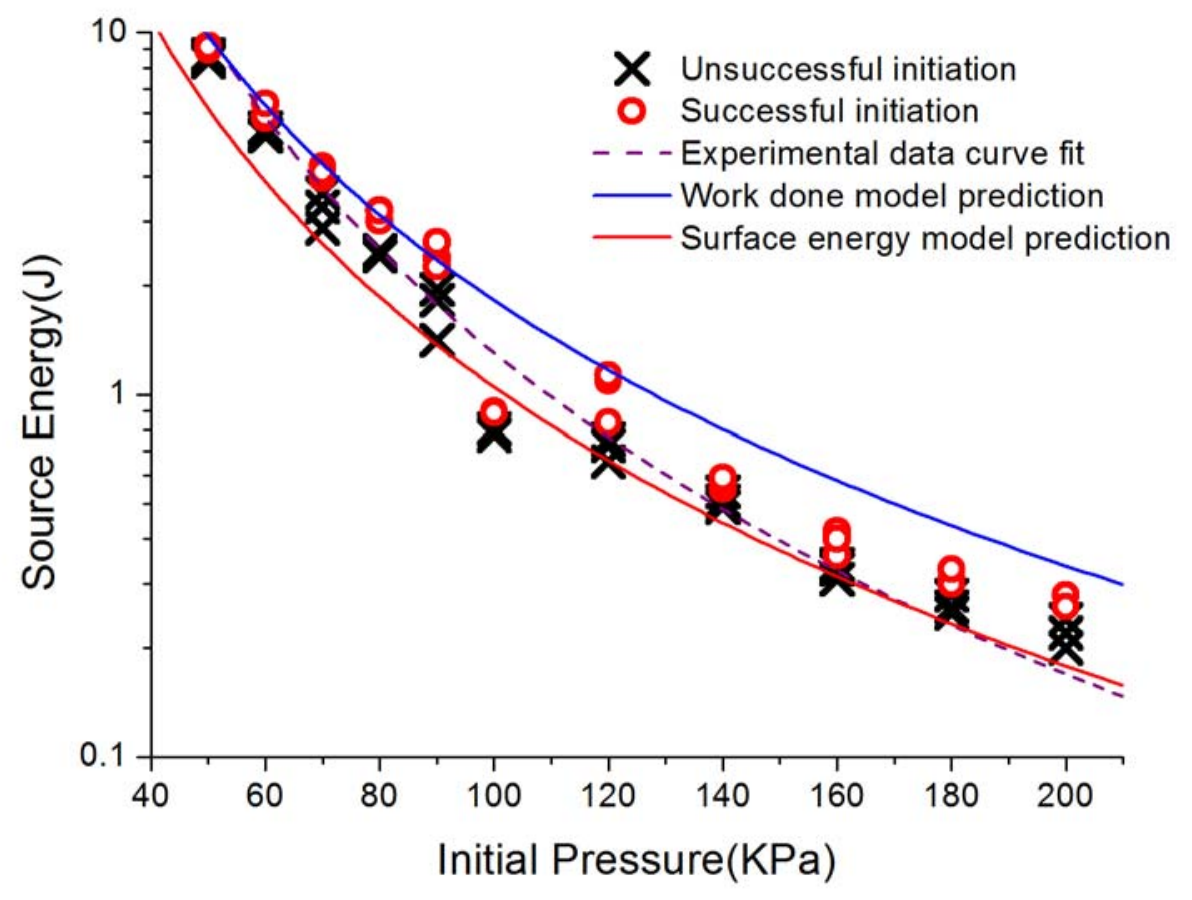

Fig. 4 


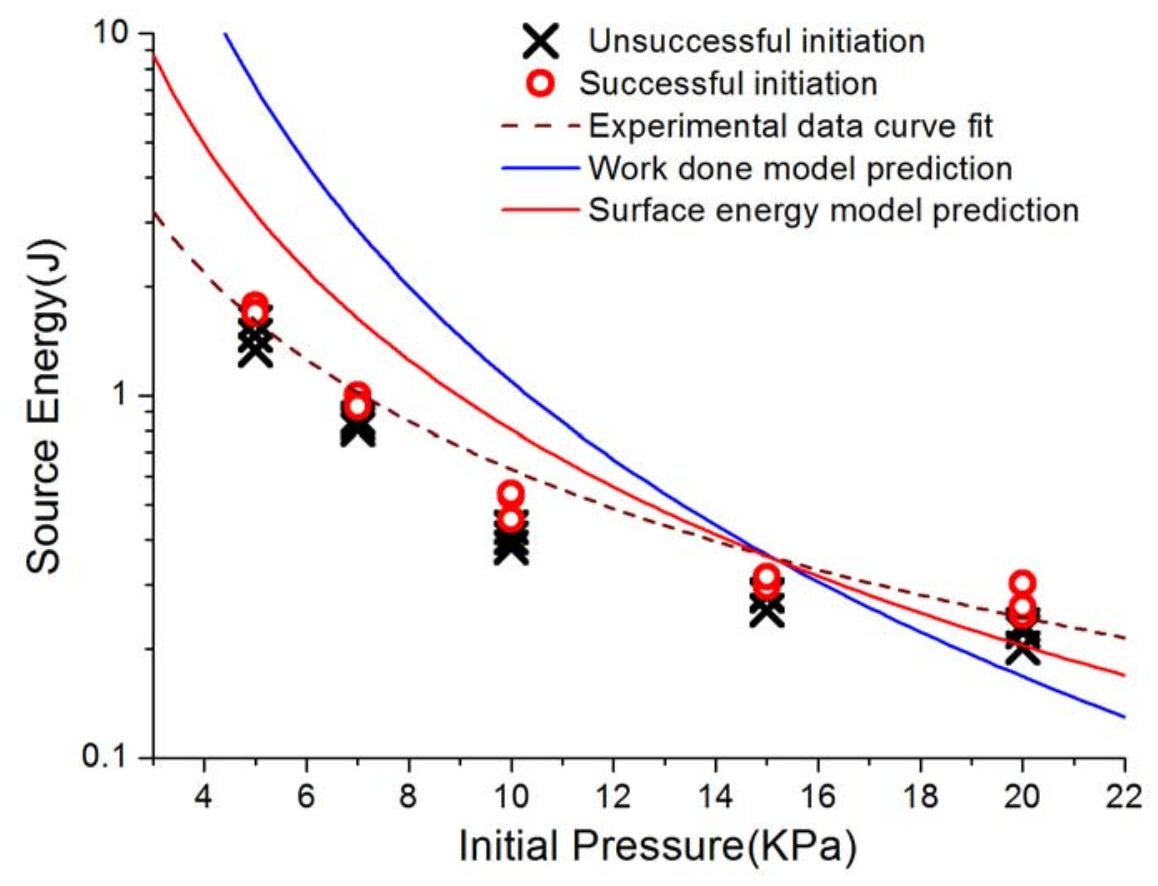

Fig. 5 


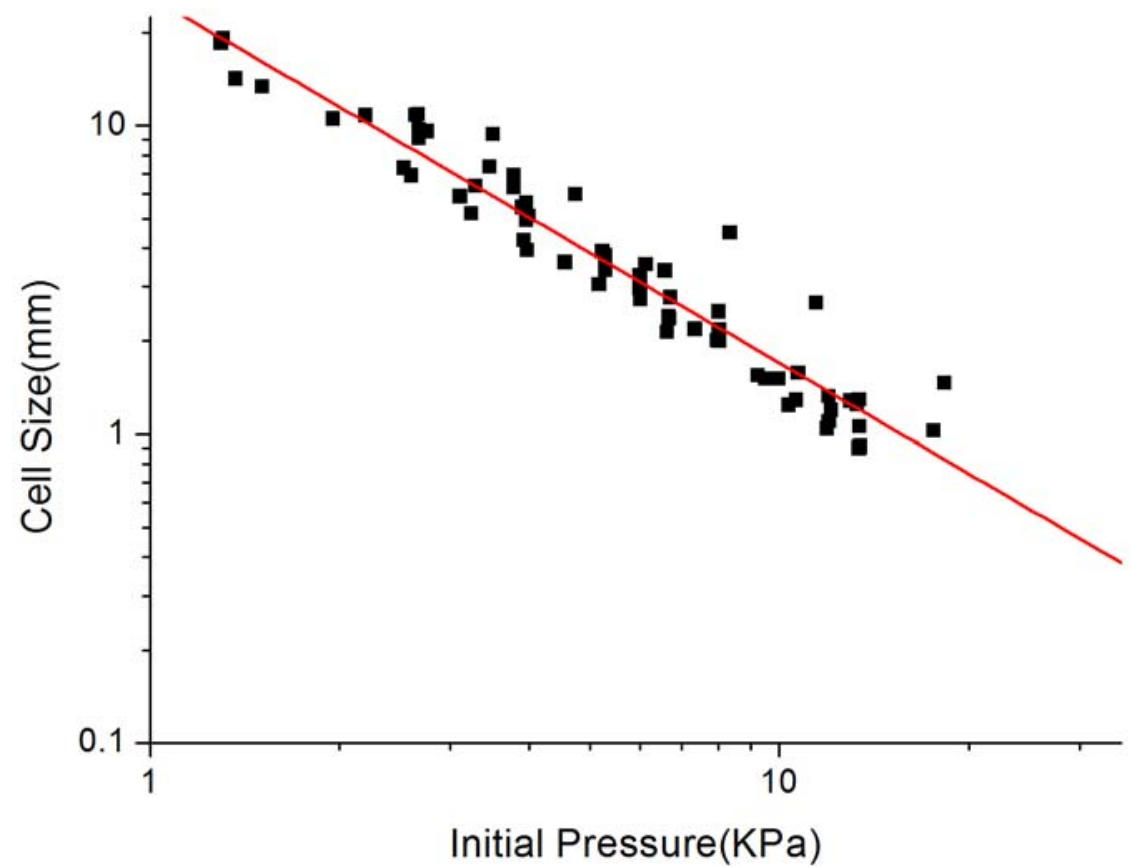

Fig. 6 